\title{
Gold nanobeacons: a potential nanotheranostics platform
}

\author{
"This early detection capability, coupled with the high level of information that can \\ be retrieved, bring additional data to clinicians, enabling early cancer staging so that \\ it can be treated appropriately."
}

Keywords: cancer therapy $\bullet$ gold nanobeacons $\bullet$ gold nanoparticles $\bullet$ nanodiagnostics - nanotheranostics

\section{Nanotheranostics}

Nanotechnology offers a plethora of tools for addressing disease diagnostics and treatment. This is particularly true when we look at cancer, in which nanoscale-based strategies have been proposed for tackling the increasing need for both accurate molecular characterization and treatment strategies. In addition, nanotechnology has prompted the development of new targeted imaging agents that are capable of the specific identification of cancer cells and the simultaneous visualization of several molecular markers (e.g., staging and typing) that would otherwise be undetectable through conventional imaging. The capability to detect specific cancerous or even precancerous cells before they have made a visible change to a tissue without the need for invasive procedures might eliminate the need for biopsies, thus reducing patient distress and healthcare costs. Some of these nanodevices allow for preventative action via the monitoring of predictive molecular changes with increased sensitivity and selectivity. This early detection capability, coupled with the high level of information that can be retrieved, bring additional data to clinicians, enabling early cancer staging so that it can be treated appropriately.

The potential of nanotechnology in medicine is not limited to diagnostics. In fact, a multitude of novel nanoformulations have been proposed for the development of multifunctional therapeutic nanodevices that are capable of bypassing biological barriers, selectively targeting cancer cells and delivering traditional or novel anticancer drugs directly to the malignant cell. In this way, lower doses of therapeutic agents may be conveyed to the cancerous tissues with high selectivity, decreasing the exposure of healthy tissues to the deleterious effects of conventional chemotherapy. Another huge advantage of these novel nanocarriers is their protective effect upon the cargo, be they chemical agents, nucleic acid molecules (e.g., siRNAs or antisense oligonucleotides) or peptides, preventing their degradation before reaching their target. Additional modifications may be added to the therapeutic platform in order to enhance cancer cell uptake, offering tight time and spatial control of biodistribution and release. Nanotechnology has put forward several alternative strategies for overcoming the limited therapeutic outcomes for advancedstage malignancies. These strategies largely revolve around the global concept of precision medicine, where nanotheranostics generate novel and effective assessments of a specific target cancer, the involvement of individual metabolic and regulatory constraints and the real-time follow-up of the therapeutic progress.

The diagnostic and therapeutic capabilities of nanomedicine formulations have been combined and used in recent years for theranostic approaches, in which cancer diagnosis, therapy and real-time monitoring are

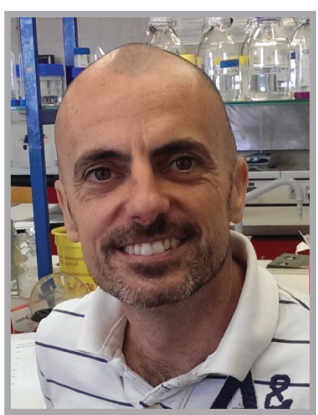

Pedro Viana Baptista

Nanomedicine@FCT, Department of Life Sciences, Faculdade de Ciências e Tecnologia, Universidade Nova de Lisboa, 2829-516 Caparica, Portugal

Tel.: +351212948530

Fax: +351212948530 pmvb@fct.unl.pt 
united in one formulation. Nanotheranostic approaches provide the ability to monitor both disease progression and therapeutic outcomes in real time [1,2]. Numerous types of nanocarriers have been developed for theranostic approaches, including polymer conjugations, dendrimers, micelles, liposomes, metal and inorganic nanoparticles, carbon nanotubes and nanoparticles of biodegradable polymers [2].

\section{Gold nanoparticles as platforms for nanotheranostics}

Among the numerous nanoformulations that have been proposed for theranostics applications, gold nanoparticles (AuNPs) have been of particular interest due to their unique properties, such as: ease of synthesis in a variety of sizes and shapes, conferring them with striking spectral properties (e.g., intense light absorption, bright colors and scattering); high photothermal conversion rates and photostabilities; colloidal stability; biocompatibility; simple ligand conjugation chemistry; and increased molecule loading per particle $[3,4]$.

AuNPs have long been proposed as tags for molecular detection and characterization protocols for in vitro applications. In terms of in vivo applications, AuNPs have been shown to be able to carry therapeutically active compounds, to be suitable for surface functionalization with multiple targeting moieties with high affinity and specificity for cancer cells and to show a high capability to accommodate therapeutic and imaging agents simultaneously, turning them into powerful theranostic platforms [5].

\section{"Gold nanoparticles can be used as powerful imaging agents for the imaging-based diagnostics of cancer cells both in vitro and in vivo."}

It is well known that the biodistribution and pharmacokinetics of AuNPs depend on properties such as size, shape, charge and surface coating. For systemic delivery, AuNPs must travel through the bloodstream until they can extravasate and passively target tumors via the enhanced permeability and retention effect. However, AuNPs need to escape the circulating plasma proteins - opsonins - that bind to foreign small molecules and microorganisms in order to remove them from the circulation through the mononuclear phagocyte system. PEGylation also serves to camouflage AuNPs by preventing the adsorption of the proteins and molecules that are involved in phagocytic uptake [6]. AuNPs can also actively target tumors through cancer-related receptors using monoclonal antibodies, peptides/proteins (e.g., cell internalization peptides, transferrin and EGF), folic acid, carbohydrates and DNA/RNA, depending on the desired cell target [7-9].

Due to their localized surface plasmon resonance, AuNPs in the size range of approximately $40 \mathrm{~nm}$ show an absorption cross-section that is five orders of magnitude higher than that of conventional absorbing dyes, without suffering from photobleaching. In the same way, the light scattering magnitude of $80-\mathrm{nm}$ AuNPs is five orders higher than those of strongly fluorescent dyes [10]. This enhanced light absorption and scattering lies at the basis of a number of biomedical applications, including photothermal therapy and various image-based detection techniques. Either due to their greatly enhanced radiative properties, electromagnetic field enhancement or their amenity for conjugation with contrast agents, AuNPs can be used as powerful imaging agents for the imaging-based diagnostics of cancer cells both in vitro and in vivo. Here, we will focus on the in vivo applications (for further reviews of AuNPs for in vitro diagnostics, please refer to $[11,12]$ and the references therein).

Multifunctional hybrid AuNPs have been developed for combined multimodal imaging (diagnostics) and therapy. Among the possible imaging modalities using AuNPs, we may include light scattering imaging, surface-enhanced Raman spectroscopy, photoacoustic imaging, $\mathrm{x}$-ray computed tomography, two-photon fluorescence imaging and MRI [13].

\section{Gold nanobeacons as nanotheranostics platforms}

One of the proposed nanotheranostics platforms is the combination of fluorescence-labeled antisense oligonucleotides and the localized surface plasmon resonance properties of AuNPs (i.e., quenching of fluorescence) - the so-called 'gold nanobeacons'. This system has been proposed as a platform for gene-silencing protocols [14,15]. A gold nanobeacon is a stem-loop ssDNA oligonucleotide functionalized with a fluorophore at one end and a AuNP (quencher) at the other end. In the absence of the complementary target (native form), the stem-loop structure is closed, forcing the fluorophore and AuNP into close proximity, resulting in fluorescence quenching; a complementary target to the loop sequence is capable of specifically hybridizing to the hairpin, forming a double-stranded structure that opens the beacon, which spatially parts the fluorophore and AuNP and fluorescence is restored [15]. By adjusting the length of both the stem and the loop, it is possible to tune the response of the nanobeacon to different cellular/tissue conditions. Fluorescence monitoring enables quantitative assessment of the gold nanobeacons' conformation changes, which 
is suitable for functional analysis under the desired conditions (e.g., real-time monitoring of DNA/RNA cleavage by enzymes, DNA/RNA-protein interactions and in vitro transcription monitoring in real time) [15]. The gold nanobeacon strategy allows for a straightforward evaluation of gene-silencing efficacy, since the fluorescence output is proportional to the number of targets being silenced. In this way, it is possible to ascertain effective therapeutic gene silencing without invasive or disruptive approaches. Additional information related to the localization of the silencing event may also be retrieved. Because the silencing moiety is bonded to the AuNPs, the antisense oligonucleotide shows an increased halflife in the presence of nucleases, since it has been shown that AuNPs confer considerable protection against enzymatic degradation $[16,17]$. What is more, the PEGylated AuNPs are extremely stable under physiological conditions and will be able to evade macrophage clearance, thus increasing their in vivo efficacy [6,13]. Perhaps one of the most relevant features of such a design is the capability conveyed by the AuNPs to act as efficient transfection agents in aqueous media (i.e., the AuNPs act as carriers or vectors for the silencing moiety and confer protection while being an active part of the imaging and tracking of the events). Another relevant characteristic is that these gold nanobeacons show no considerable toxicity at the concentrations required for effective silencing [18]. The versatility of the gold nanobeacon platform for targeting different gene regulation pathways under the same molecular design is one of its most valuable features. Indeed, this system has been shown to be effective at silencing specific genes or targeting endogenous (or exogenous) silencing machinery (i.e., the nanobeacon is effective at silencing endogenous miRNAs). This is of the utmost relevance due the acknowledged involvement of miRNAs in the regulation of specific gene expression in numerous disease states, such as cancer.

This strategy defines the way of using these gold nanoconjugates for the detection (diagnostics) and, at the same time, the inhibition of specific genes (therapy). The application of such an approach in vivo will require the use of other fluorophores and/or imaging tags, such as molecules that are of use in different regions of the spectra. For example, this molecular architecture may be applied to near-infrared fluorophores or adapted to Raman fingerprinting (e.g., using surface-enhanced Raman spectroscopy modulation by the AuNPs). Such modifications require further studies and characterization, mainly in in vivo models, before they can be applied as effective nanotheranostics platforms in the clinic.
Despite the large number of reports in the literature on systems for nanotheranostics, there are only a few such systems that have reached in vivo studies and actually made it into clinical trials. Among these, AuNP-based systems are no different. From the plethora of proposed platforms being developed and evaluated, the majority still lack two essential assessments: toxicity/biosafety assessment and subsequent evaluation of clinical efficacy. The lack of standards and specific regulations by the official and responsible offices (e.g., the EMA and the US FDA) has hampered further developments in the field. It is noteworthy that the bulk of the systems under study and eventual translation into the clinic are focused in cancer diagnostics and treatment. This is probably due to the fact that the benefits in these cases/patients clearly outweigh the eventual issues of efficacy. Gold nanobeacons show enormous promise as vectors for gene silencing in theranostics applications. However, no nanomaterial comes without limitations. Indeed, although gold nanobeacons may be considered biocompatible, a number of studies have reported that the toxicity of AuNPs alone depends on their size, shape and surface coating, among other factors. Some other limitations include the fact that AuNPs are nonbiodegradable (resulting in possible bioaccumulation in a number of tissues and organs). However, perhaps the most significant challenge is to comprehend the overall behavior of the gold nanobeacons in complex organisms, namely in terms of their biodistribution, pharmacokinetics and biocompatibility, and therefore detailed characterization studies must be conducted. In addition, long-term toxicity studies are definitely required in order to understand the fate of these platforms in the organism and to understand the mechanisms by which AuNPs, and gold nanobeacons in particular, interact with biological systems.

\section{Acknowledgements}

The author would like to thank A Fernandes for fruitful discussions.

Financial \& competing interests disclosure

The author acknowledges financial support from Centro de Investigação em Genética Molecular Humana-Fundação para a Ciência e Tecnologia/Ministérios da Ciências e do Ensino Superior (CIGMH-FCT/MCES). The author has no other relevant affiliations or financial involvement with any organization or entity with a financial interest in or financial conflict with the subject matter or materials discussed in the manuscript apart from those disclosed.

No writing assistance was utilized in the production of this manuscript. 


\section{References}

Papers of special note have been highlighted as:

• of interest; $\bullet$ of considerable interest

1 Lammers T, Kiessling F, Hennink WE et al.

Nanotheranostics and image-guided drug delivery: current concepts and future directions. Mol. Pharm. 7(6), 1899-1912 (2010).

2 Muthu MS, Leong DT, Mei L et al.

Nanotheranostics - application and further development of nanomedicine strategies for advanced theranostics. Theranostics 4(6), 660-677 (2014).

- Inclusive discussion on the current state of the art of nanotheranostics applications.

3 Almeida JP, Figueroa ER, Drezek RA. Gold nanoparticle mediated cancer immunotherapy. Nanomedicine 10(13), 505-514 (2014).

4 Dreaden EC, Alkilany AM, Huang X et al. The golden age: gold nanoparticles for biomedicine. Chem. Soc. Rev. 41(7), 2740-2779 (2012).

5 Rahman M, Ahmad MZ, Kazmi I et al. Advancement in multifunctional nanoparticles for the effective treatment of cancer. Expert Opin. Drug Deliv. 9(4), 367-381 (2012).

6 Cavadas M, González-Fernández A, Franco R. Pathogenmimetic stealth nanocarriers for drug delivery: a future possibility. Nanomedicine 7(6), 730-743 (2011).

7 Sperling RA, Parak WJ. Surface modification, functionalization and bioconjugation of colloidal inorganic nanoparticles. Philos. Trans. A Math. Phys. Eng. Sci. 368(1915), 1333-1383 (2010).

- Comprehensive discussion of funcional moiety modification on nanoparticle surfaces.

8 Conde J, Doria G, Baptista P. Noble metal nanoparticles applications in cancer. J. Drug Deliv. 2012, 751075 (2012).

9 Kim D, Friedman AD, Liu R. Tetraspecific ligand for tumortargeted delivery of nanomaterials. Biomaterials 35(23), 6026-6036 (2014).
10 Acuna GP, Bucher M, Stein IH et al. Distance dependence of single-fluorophore quenching by gold nanoparticles studied on DNA origami. ACS Nano 6(4), 3189-3195 (2012).

- Comprehensive study of distance-dependent fluorescence modulation by gold nanoparticles.

11 Khlebtsov N, Bogatyrev V, Dykman L et al. Analytical and theranostic applications of gold nanoparticles and multifunctional nanocomposites. Theranostics 3(3), 167-180 (2013).

- Discusses the analytical and theranostics applications of gold nanoparticles.

12 Baptista PV. Could gold nanoprobes be an important tool in cancer diagnostics? Expert Rev. Mol. Diagn. 12(6), 541-543 (2012).

13 Cabral RM, Baptista PV. The chemistry and biology of gold nanoparticle-mediated photothermal therapy: promises and challenges. Nano LIFE 3(3), 1330001 (2013).

14 Rosa J, Conde J, de la Fuente J et al. Gold-nanobeacons for real-time monitoring of RNA synthesis. Biosens. Bioelectron. 36(1), 161-167 (2012).

15 Conde J, Rosa J, de la Fuente JM et al. Gold-nanobeacons for simultaneous gene specific silencing and intracellular tracking of the silencing events. Biomaterials $34(10)$, 2516-2523 (2013).

-• Describes the chemistry and functionality of gold nanobeacons.

16 Conde J, de la Fuente JM, Baptista PV. In vitro transcription and translation inhibition via DNA functionalized gold nanoparticles. Nanotechnology 21, 505101 (2010).

17 Barnaby SN, Lee A, Mirkin CA. Probing the inherent stability of siRNA immobilized on nanoparticle constructs. Proc. Natl Acad. Sci. USA 111(27), 9739-9744 (2014).

18 Conde J, Larguinho M, Cordeiro A et al. Gold-nanobeacons for gene therapy: evaluation of genotoxicity, cell toxicity and proteome profiling analysis. Nanotoxicology 8(5), 521-532 (2013). 